\title{
EL PROCESO DE TRANSFORMACIÓN HACIA UN BOLETÍN DE EVALUACIÓN CUALITATIVO E INDIVIDUAL EN EDUCACIÓN INFANTIL MEDIANTE INVESTIGACIÓN-ACCIÓN PARTICIPATIVA
}

The process of transformation towards a qualitative and individual evaluation bulletin in childhood education through participatory action-research

O processo de transformação em direção a um boletim de avaliação qualitativo e individual na educação pré-escolar, através da investigação-ação participativa

\section{Raúl A. Barba-Martín (1) \\ Carlos Sanz Simón (2)}

\begin{abstract}
Este estudio se ha llevado a cabo dentro del proyecto de $\mathrm{I}+\mathrm{D}+\mathrm{i}$ : "La competencias docentes en la formación inicial del profesorado de educación física". Convocatoria de noviembre de 2013 del Programa Estatal de Investigación, Desarrollo e Innovación Orientada a los Retos de la Sociedad, en el marco del Plan Estatal de Investigación Científica y Técnica y de Innovación 2013-2016. Referencia: EDU 201342024-R. Duración: 3 años (2014-2016). A su vez, se ha realizado gracias a la concesión de un contrato predoctoral de personal investigador en formación, cofinanciado por la Universidad Complutense de Madrid y el Banco Santander (Convocatoria CT17/17-CT18/17).
\end{abstract}

(1) Universidad de Valladolid, España. Teléfono: +34 921112321. Correo electrónico: raulalberto.barba@uva.es

(2) Universidad Complutense de Madrid, España. Teléfono: +34 913946139. Correo electrónico: csa02@ucm.es

\section{Resumen}

Este estudio analiza en qué medida un proceso de formación permanente del profesorado basado en investigación-acción participativa parece incidir en la transformación de los boletines de evaluación (o informativos) que se entregan a las familias en la etapa de Educación Infantil. La investigación se lleva a cabo dentro de Proyecto de Innovación Docente cuya finalidad es fomentar la innovación educativa en las escuelas, dirigida a transformar sus prácticas de aula por otras orientadas al aprendizaje inclusivo y participativo. Los resultados parecen indicar que los proyectos de formación permanente basados en investigación-acción participativa conllevan un cambio en el pensamiento de las docentes, que les hace replantearse sus prácticas educativas, incluidos los instrumentos de evaluación.

Palabras clave: Formación permanente; investigación-acción participativa; evaluación compartida; boletín; Educación Infantil

El proceso de transformación hacia un boletín de evaluación cualitativo e individual en Educación Infantil mediante investigación-acción participativa 


\begin{abstract}
This study analyzes the extent to which a process of ongoing teacher training based on participatory action research seems to influence the transformation of the evaluation (or information) bulletins that are delivered to families in the early childhood education stage. The research takes place within the Teaching Innovation Project for educational innovation in schools, aimed at classroom practices by others oriented to inclusive and participatory learning. The results seem to indicate the projects of permanent training in participatory action-research entail a change in the thinking of the teachers, reconsidering their own educational practices, including the evaluation instruments.
\end{abstract}

Keywords: Ongoing education; participatory action-research; shared evaluation; bulletin; Early Childhood Education

\title{
Resumo
}

Este estudo analisa em que medida um processo de formação permanente de professores baseado na investigação-ação participativa parece influenciar a transformação dos boletins de avaliação (ou informação) que são entregues às famílias na Educação PréEscolar. A investigação é realizada no âmbito do Projeto de Inovação Docente, cujo objetivo é promover a inovação educativa nas escolas, visando transformar as suas práticas na aula com outras centradas na aprendizagem inclusiva e participativa. Os resultados parecem indicar que os projetos de formação contínua, baseados em investigação-ação participativas, levam a uma mudança no pensamento dos professores, o que os faz repensar as suas práticas educativas, incluindo os instrumentos de avaliação.

Palavras-chave: formação permanente; investigação-ação participativa; avaliação partilhada; boletim; Educação Pré-Escolar

\section{Introducción}

Uno de los objetivos de la evaluación en la etapa de Educación Infantil debe ser ofrecer a los adultos instrumentos que les permitan comprender el proceso de aprendizaje de sus hijos e hijas (Díaz \& Velasco, 2018). El principal instrumento utilizado para esta labor es el boletín informativo de evaluación que los docentes entregan a los familiares al finalizar el trimestre. Sin embargo, su objetivo a veces no se consigue plenamente. La 
obligación por parte de algunos centros educativos de aplicar boletines de evaluación basados en la consecución o no de ciertos ítems -Educación Infantil- o en la calificación -Educación Primaria y Secundaria-, lleva al profesorado a la asunción y continuación de praxis tradicionales en la evaluación centradas únicamente en el resultado final.

La formación permanente se erige como una oportunidad para adquirir una conciencia crítica y transformar los procesos de evaluación (Barba-Martín \& López-Pastor, 2017). La reflexión docente sobre la propia práctica es uno de los procedimientos más importantes para la formación del profesorado (Barba, González-Calvo, \& BarbaMartín, 2014; Schön, 1988). Cuando estos procesos de reflexión se realizan sobre-laacción (alejados de la acción en tiempo y espacio) (Zeichner \& Liston, 1996) suponen la adquisición de un pensamiento crítico, necesario para procurar transformaciones educativas y sociales (Kincheloe, 2012; Giroux, 1990). Esta transformación del pensamiento y de las prácticas educativas es más fuerte si las reflexiones se realizan de forma colaborativa a través de investigación-acción (Stringer, 2014; Zeichner, 2010).

De esta forma, el objetivo de nuestra investigación es analizar si la formación permanente a través de investigación-acción participativa ayuda al profesorado de Educación Infantil a transformar sus prácticas de evaluación. En esta comunicación abordaremos el caso concreto de un instrumento de evaluación, los boletines informativos que son entregados a las familias al acabar el trimestre.

\section{Método}

La investigación ha tenido lugar en un Proyecto de Innovación Docente de la Facultad de Educación de Segovia que realiza una formación permanente sobre educación inclusiva a través de investigación-acción participativa (PID-FPEIAP). El grupo constituido para el Proyecto es multinivel, se sirve del trabajo conjunto entre profesorado universitario, docentes de escuela y estudiantes de Magisterio.

El PID-FPEIAP se basa en el modelo de investigación-acción participativa (I-A-P) de Greenwood y Levin (2007). Para estos investigadores la I-A-P es un proceso participativo en el que los investigadores trabajan y comparten saberes e ideas con los "dueños del problema" y el conocimiento se crea de forma lateral entre los participantes, a través de la reflexión compartida sobre sus experiencias (Hargreaves \& 
Shirley, 2009). Su finalidad es la transformación de las prácticas educativas y sociales de los participantes.

Los datos fueron recogidos mediante varias técnicas e instrumentos: (a) entrevistas individuales; (b) grupo de discusión; (c) observaciones participantes; y (d) cuestionarios cualitativos.

\section{Resultados y discusión}

Los resultados muestran que antes de su participación en el PID-FPEIAP el profesorado utilizaba únicamente los boletines de evaluación que el centro les obligaba a rellenar. Esta es una postura común, pues la formación inicial resulta insuficiente para la adquisición de una conciencia crítica que permita a los docentes replantearse los modelos de evaluación preestablecidos, lo cual conlleva la reproducción de los métodos de evaluación que utilizaron con ellos cuando eran alumnos (Hamodi, López-Pastor, \& López-Pastor, 2017; Pérez-Pueyo, Hortigüela, \& Gutierrez, 2016).

Estos boletines de evaluación consistían en escalas gráficas, uno de los instrumentos más utilizados para la evaluación en Educación Infantil (López-Pastor \& Pérez-Pueyo, 2017). Las maestras aludían a que la utilización de estas escalas como boletines de evaluación solo les permitía proporcionar a los familiares una información final y cerrada, pero no desarrollar el proceso de aprendizaje del alumnado. Esto se debía a que estos instrumentos contaban con unos ítems cerrados y estandarizados para todo el alumnado del centro, basados en aspectos como el comportamiento, la higiene o el aprendizaje de forma genérica y donde el profesorado únicamente podía informar a los familiares de si el alumnado lo había conseguido, estaba en proceso o no lo había conseguido.

Las maestras transformaron estos boletines a raíz de su participación en el PID-FPEIAP. Las docentes afirman que en el Proyecto adquirieron una comprensión crítica de la educación $\mathrm{y}$, como consecuencia, comenzaron a replantearse todas sus prácticas educativas, incluida la evaluación y los boletines.

Actualmente realizan dos boletines informativos, el pedido desde la institución y uno cualitativo, en el que redactan ampliamente el proceso de aprendizaje del alumnado a lo largo de todo el trimestre. Las maestras afirman que no ha sido un proceso fácil, ya que la presión desde la institución por cumplir con el boletín informativo con escala es obligatoria y, por tanto, el nuevo boletín les supone un trabajo doble. El cuál afirman es 
compensado por cumplir con su actual idea acerca de la educación y por la valoración positiva de las familias hacia este nuevo boletín informativo.

\section{Conclusiones}

La investigación expone la consecución de un proceso de formación a través de I-A-P que ha llevado a sus participantes a transformar sus boletines de evaluación finales. Este proceso se inicia con el replanteamiento de sus prácticas de evaluación por parte de los participantes. Al haber transformado previamente sus prácticas de aula en pro de aprendizajes inclusivos y la participación familiar (Barba-Martín, Barba, \& MartínezScott, 2016; Barba-Martín, González-Cavo, \& Martínez-Scott, 2018), los participantes denotaron que estas prácticas y aprendizajes que llevaban a cabo no eran consecuentes con sus prácticas de evaluación y decidieron cambiarlas. Así, transformaron los boletines finales de evaluación que les entregaban a las familias y que se basaban en la consecución de ítems cerrados, por un boletín cualitativo en el que exponen el proceso de aprendizaje individualizado de cada alumno.

Los resultados muestran el inicio de un camino hacia procesos de evaluación formativa y compartida capaces de involucrar realmente a las familias y el alumnado. Ampliar los boletines de evaluación para procurar una mayor comprensión por parte de las familias es un primer paso significativo para avanzar hacia la participación conjunta en la evaluación.

\section{Referencias}

Barba, J. J., González-Calvo, G., \& Barba-Martín, R. A. (2014a). El uso de los diarios del profesorado como instrumento de reflexión sobre la acción. Revista Española de Educación Física y Deportes, REEFD, 405, 55-63.

Barba-Martín, R.A., Barba, J. J., \& Martínez Scott, S. (2016). La formación continua colaborativa a través de la investigación-acción. Una forma de cambiar las prácticas de aula. Contextos Educativos, 19, 161-175

Barba-Martín, R. A., González-Calvo, G., \& Martínez-Scott, S. (2018). El papel de una maestra en la integración de una alumna sordociega en la comunidad educativa. Educar, 54(1), 83-99.

Barba-Martín, R. A., \& López-Pastor, V. M. (2017). La transformación de los procesos de evaluación en educación infantil mediante la formación permanente a través 
de la investigación-acción. Revista Infancia, Educación y Aprendizaje, 3(2), 674-679.

Díaz, E. C., \& Velasco, A. J. (2018). Concepciones docentes acerca de la evaluación y el boletín informativo en educación inicial. Pensamiento Educativo. Revista de Investigación Educacional Latinoamericana, 55(1), 1-17.

Giroux, H. A. (1990). Los profesores como intelectuales. Hacia una pedagogía crítica del aprendizaje. Barcelona: Paidós/MEC.

Greenwood, D. J., \& Levin, M. (2007). Introduction to action research. Social research for social change (2nd ed.). Thousand Oaks, CA: Sage.

Hamodi, C., López-Pastor, V. M., \& López-Pastor, A. T. (2017). If I experience formative assessment whilst at University will I put it into practice later as a teacher? Formative and shared assessment in Initial Teacher Education (ITE). European Journal of Teacher Education, 40(2), 171-190.

Hargreaves, A., \& Shirley, D. (2009). The fourth way. The inspiring future for educational change. Thousand Oaks, CA: Corwin

Kincheloe, J. L. (2012). Teachers as researches. Qualitative inquiry as a path to empowerment ( $4^{\mathrm{a}}$ ed.). Wiltshire: Routledge.

López-Pastor, V.M., \& Pérez-Pueyo, A. (coords.) (2017). Evaluación formativa y compartida en educación: experiencias en todas las etapas educativas. León: Universidad de León.

Pérez Pueyo, A., Hortigüela, D., \& Gutierrez, C. (2016). Reflexión sobre la evaluación en la formación inicial del profesorado en España. En búsqueda de la concordancia entre dos mundos. Infancia, Educación y Aprendizaje, 2(2), 39-75.

Schön, D. A. (1988). El profesional reflexivo. Cómo piensan los profesionales cuando actúan. Barcelona: Paidós.

Stringer, E. T. (2014). Action research (4 $4^{\mathrm{a}}$ ed.). Thousand Oaks, CA: SAGE.

Zeichner, K. M. (2010). Nuevas epistemologías en formación del profesorado. Revista Interuniversitaria de Formación del Profesorado, 68(24, 2), 123-149.

Zeichner, K. M., \& Liston, D. P. (1996). Reflective teaching. An introduction. Mahwah, NJ: Lawrence Erlbaum Associates, Inc., Publishers.

El proceso de transformación hacia un boletín de evaluación cualitativo e individual en Educación Infantil mediante investigación-acción participativa 